ARTICLE

\title{
High-performance coherent optical modulators based on thin-film lithium niobate platform
}

Mengyue Xu (1) 1, Mingbo He, Hongguang Zhang, 2,3, Jian Jian', Ying Pan', Xiaoyue Liu (D) 1, Lifeng Chen (1) 1,

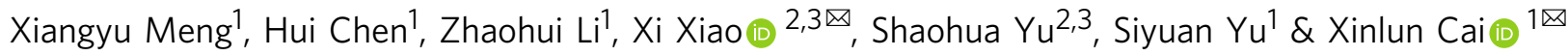

The coherent transmission technology using digital signal processing and advanced modulation formats, is bringing networks closer to the theoretical capacity limit of optical fibres, the Shannon limit. The in-phase/quadrature electro-optic modulator that encodes information on both the amplitude and the phase of light, is one of the underpinning devices for the coherent transmission technology. Ideally, such modulator should feature a low loss, low drive voltage, large bandwidth, low chirp and compact footprint. However, these requirements have been only met on separate occasions. Here, we demonstrate integrated thin-film lithium niobate in-phase/quadrature modulators that fulfil these requirements simultaneously. The presented devices exhibit greatly improved overall performance (half-wave voltage, bandwidth and optical loss) over traditional lithium niobate counterparts, and support modulation data rate up to $320 \mathrm{Gbit} \mathrm{s}^{-1}$. Our devices pave new routes for future highspeed, energy-efficient, and cost-effective communication networks.

\footnotetext{
${ }^{1}$ State Key Laboratory of Optoelectronic Materials and Technologies and School of Electronics and Information Technology, Sun Yat-sen University, 510006 Guangzhou, China. ${ }^{2}$ National Information Optoelectronics Innovation Center, China Information and Communication Technologies Group Corporation (CICT), 430074 Wuhan, China. ${ }^{3}$ State Key Laboratory of Optical Communication Technologies and Networks, China Information and Communication Technologies Group Corporation (CICT),430074 Wuhan, China. ${ }^{凶}$ email: xxiao@wri.com.cn; caixlun5@mail.sysu.edu.cn
} 
O ver decades and across all levels of optical networks, the global internet traffic has experienced continuous growth at an enormous rate ${ }^{1,2}$. To keep up with this everincreasing demand, the digital coherent transmission technology has been introduced for long-haul communication links and is allowing networks to approach the maximum achievable capacity of optical fibres, known as the Shannon limit ${ }^{3-7}$. This technology is now expected to penetrate into the rapidly growing, capacityhungry short reach links, such as metro and data-centre interconnects, where an in-phase/quadrature (IQ) modulator must be operated in a small space, while featuring low loss, low drive voltages, and large bandwidths ${ }^{8-10}$. For nearly a decade, IQ modulators based on low-index-contrast lithium niobate $\left(\mathrm{LiNbO}_{3}, \mathrm{LN}\right)$ waveguides have been the mainstay for generating the advanced modulation formats ${ }^{11-14}$. Although these modulators have enjoyed tremendous success in long-haul coherent networks, their performance is already reaching a limit because the low-index-contrast LN waveguides cannot support them. To date, the off-the-shelf LN-based IQ modulators are still bulky and power-consuming with a moderate half-wave voltage $\left(V_{\pi}\right)$ of $3.5 \mathrm{~V}$ requiring devices of at least $5 \mathrm{~cm}$, and with little hope for further improving the electric-optic (EO) bandwidth (typically around $35 \mathrm{GHz}$ ), which limits their practical short reach applications.

Tremendous efforts have been made to realise small-footprint and high-performance IQ modulators in various material platforms, including silicon ( $\mathrm{Si}$ ), indium phosphide ( $\mathrm{InP})$, polymers, and plasmonics ${ }^{15-32}$. Although these platforms normally offer the advantages of compact footprints and large bandwidths, each type of modulator has its limitations, including large $V_{\pi}(\mathrm{Si})$, high optical loss (plasmonics), nonlinear response (InP), or doubts over long-term stability (polymer). The development of an ideal IQ modulator that possesses all the desired characteristics simultaneously remains a challenge, mainly due to limitations from underlying materials.

LN-on-insulator (LNOI) has recently emerged as an appealing material platform for compact and high-performance modulators, on which high-contrast waveguides with strong optical confinement can be formed by simply etching the device layer of an LNOI wafer ${ }^{33-46}$. This approach unlocks new levels of performance and scales in LN modulators because it overcomes the fundamental voltage-bandwidth-size trade-off in conventional low-index-contrast LN modulators. Recently, LNOI-based Mach-Zehnder modulators (MZMs) with low drive voltages and ultrahigh EO bandwidths, which significantly outperform their conventional counterparts, have been demonstrated (see Supplementary Note 1).

Here, we demonstrate the IQ modulator based on the LNOI platform, which is capable of encoding signals with advanced modulation formats, such as quadrature phase-shift keying (QPSK) and quadrature amplitude modulation (QAM) signals. The proposed device features low optical loss, low $V_{\pi}$, ultrahigh EO bandwidth, and much smaller footprint than the conventional LN counterpart. Furthermore, QPSK modulation up to $220 \mathrm{Gbit} \mathrm{s}^{-1}$ (110 Gbaud), and 16 QAM modulation up to 320 Gbit $\mathrm{s}^{-1}$ (80 Gbaud), are successfully demonstrated.

\section{Results}

Device design. Figure 1a illustrates the schematic of the proposed device. The LNOI-based IQ modulator is constructed by nesting two parallel travelling-wave MZMs as I and Q components, respectively. The optical power splitters/combiners are implemented by $1 \times 2$ multimode interference (MMI) couplers (see Supplementary Note 2, Supplementary Figs. 1 and 2). Both I and Q MZMs are balanced travelling-wave modulators with ground-signal-ground (GSG) microelectrodes, where the two LN arms lie in the gaps of the ground and signal electrodes. (Fig. 1b) The device operates in a single-drive push-pull configuration, so that applied microwave fields induce phase shifts with an equal magnitude but opposite sign in both arms, leading to nearly chirp-free modulation. Two thermo-optic (TO) phase shifters (DC1 and DC2) are employed to control the modulation bias points of the I and Q branches independently. (Fig. 1c) For QPSK and QAM modulation, the bias points need to be set at null points. A third TO phase shifter (DC3) introduces a static $\pi / 2$ phase shift between the modulated signals from the two subMZMs, which puts them in quadrature to each other. To couple light in and out of the device, amorphous silicon/LN hybrid grating couplers are used for transverse-electric (TE) mode coupling 47 .

The cross-section of the LN waveguides, together with the travelling-wave electrodes, are optimised to achieve a low $V_{\pi}$ and a large EO bandwidth simultaneously. The LN waveguides in the phase modulation sections have a top width $w$ of $4 \mu \mathrm{m}$, a slab thickness $s$ of $300 \mathrm{~nm}$ and a rib height $h$ of $300 \mathrm{~nm}$. The sidewall of the waveguide is tilted with an angle of $64^{\circ}$ (Fig. 1d). The lithography and etching processes were optimised to achieve smooth sidewalls, which was confirmed by atomic force microscope (AFM) measurements (see Supplementary Note 3 and Supplementary Fig. 3). The propagation loss of single-mode and 4-um-wide $\mathrm{LN}$ waveguides were measured to be $0.3 \mathrm{~dB} \mathrm{~cm}^{-1}$ and $0.15 \mathrm{~dB} \mathrm{~cm}^{-1}$, respectively (see Supplementary Fig. 4). The gap between the LN waveguides and electrodes was set to $1.5 \mu \mathrm{m}$. These parameters are devised carefully to balance the trade-off between the voltage-length product $\left(V_{\pi} L\right)$ and bandwidth-voltage ratio $\left(\mathrm{BW} / V_{\pi}\right)$, which are two key figures of merit of optical modulators (see Supplementary Note 4 and Supplementary Fig. 5). The coplanar travelling-wave electrodes are designed to simultaneously achieve broadband impedance matching, as well as velocity matching of the microwave and optical signals. The thickness of the electrodes $t$ was set to $900 \mathrm{~nm}$; the gaps between the signal and ground electrodes were set to $7 \mu \mathrm{m}$, and the widths of the signal $w_{\mathrm{s}}$ and ground $w_{\mathrm{g}}$ electrodes were set to 19.5 and 80 $\mu \mathrm{m}$, respectively (Fig. 1e).

Device characteristics. The fabricated LNOI IQ modulators with arm lengths of $7.5 \mathrm{~mm}$ and $13 \mathrm{~mm}$ are measured in detail. We first measured the direct current transmission with TO phase shifters. Figure 2a shows the TO transmission curve as a function of the applied voltage. The length of the TO phase shifter is only $160 \mu \mathrm{m}$ with a resistance of $760 \Omega$, and the required voltages for biasing at quadrature and null are 3.55 and $7.2 \mathrm{~V}$, corresponding to power dissipations of 16.6 and $68.2 \mathrm{~mW}$, respectively. Instead of the EO effect, the TO effect is used here for DC bias voltage control, offering two distinct advantages. First, the TO effect is much stronger compared to the EO effect, allowing for much compact device size. In our case, the length of the EO phase shifter would have been more than $5 \mathrm{~mm}$ for the same amount of voltage. Second, LN is well-known for its drifts in the DC bias point upon the application of a static electric field, which is a phenomenon that originates from the piezoelectric nature of the material ${ }^{11,48,49}$. This drift must be compensated by a fast feedback loop for practical use. This phenomenon is absent in TO phase shifters, and a much simpler control scheme could be used instead. To provide a comparative study, we record the output power from an LNOI MZM biased at quadrature using both TO and EO phase shifters. Figure $2 \mathrm{~b}$ shows the measured results in $30 \mathrm{~min}$, confirming that the DC bias point with the TO effect is much more stable than with the EO effect. It should be noted that the TO phase shifter consumes static power, while EO phase 

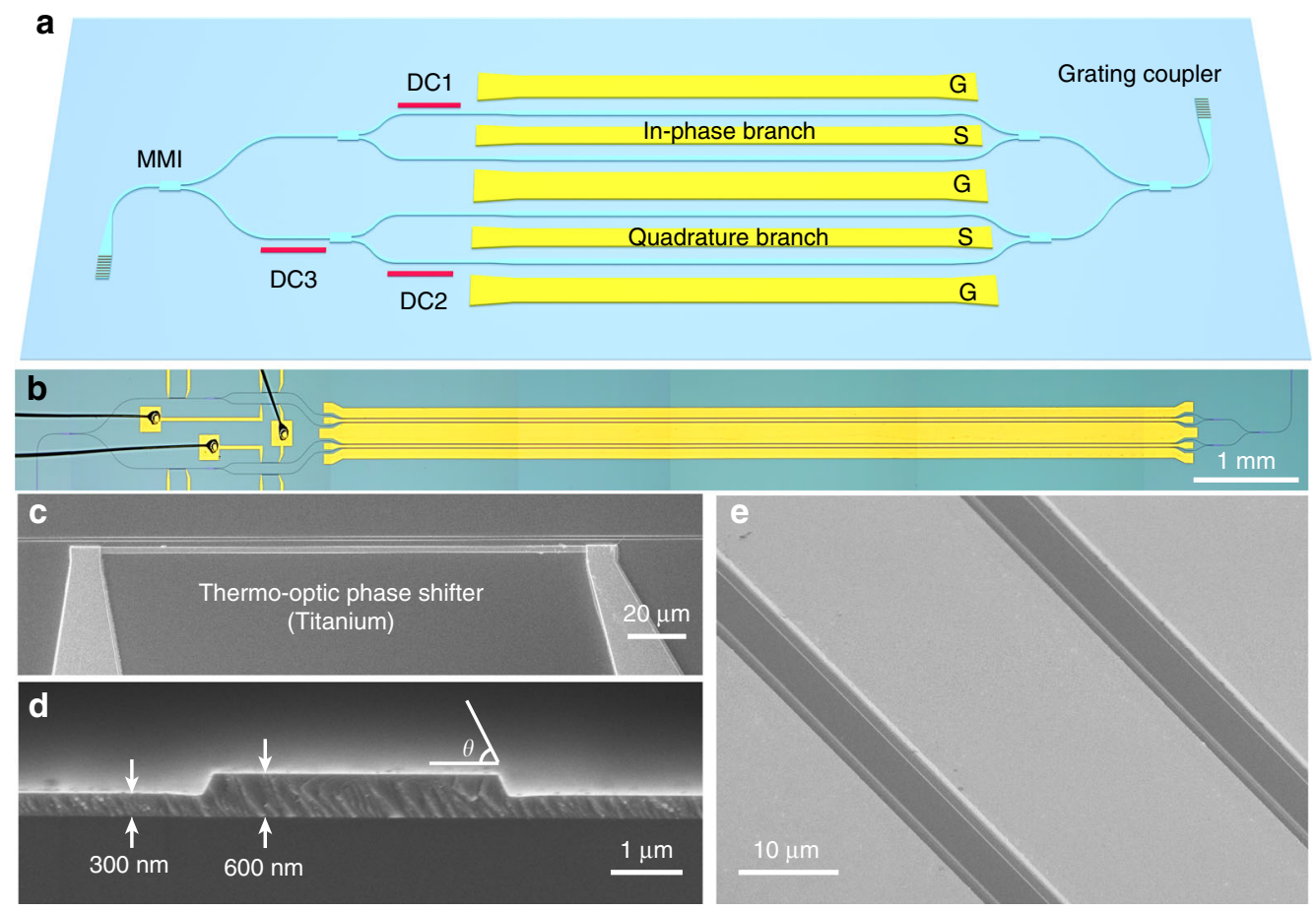

Fig. 1 IQ modulator on the LNOI platform. a Schematic of an LNOI-based IQ modulator. b Microscope image of the fabricated chip. c Scanning electron microscopy (SEM) image of the thermos-optic phase shifter. d SEM image of the cross-section of the LN waveguides. e SEM image the gold electrodes and the LN waveguides.
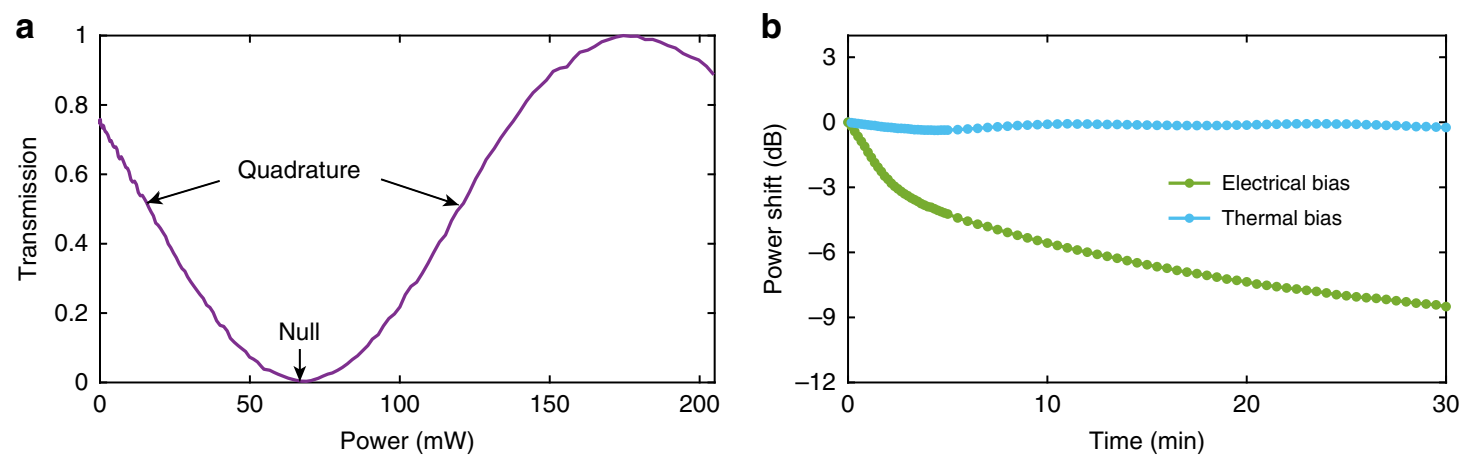

Fig. 2 TO phase shifters performance. a TO transmission curve as a function of the power dissipations. b Power shift from an MZM biased at quadrature using EO (green line) and TO (blue line) phase shifters as a function of the operating time.

shifter does not. We also fabricated an IQ modulator with EO phase shifters for further comparison (Supplementary Note 5, Supplementary Fig. 6 and Supplementary Table 1).

Next, we measured the $V_{\pi}$ for both devices with $100 \mathrm{kHz}$ triangular voltage sweeps. The measured $V_{\pi}$ for the 7.5 and 13 $\mathrm{mm}$ devices are 3.1 and $1.9 \mathrm{~V}$, corresponding to $V_{\pi} L$ of 2.3 and $2.4 \mathrm{~V} \mathrm{~cm}$, respectively (Fig. 3a, b). The inset of Fig. 3a shows the transmission of one of the sub-MZMs on a logarithmic scale, indicating a measured extinction ratio of $>25 \mathrm{~dB}$. We have fabricated more than ten devices on the same chip, and the measured extinction ratios are between 24 and $28 \mathrm{~dB}$. Figure $3 \mathrm{~b}$ shows the optical transmission at different wavelengths, showing broadband operation in the whole C-band.

We then characterised the small-signal EO bandwidth $\left(S_{21}\right.$ parameter) and electrical reflections $\left(\mathrm{S}_{11}\right)$ of the fabricated devices. For the 13-mm device, the measured 3-dB EO bandwidths of both I and Q MZMs are greater than $48 \mathrm{GHz}$ with a reference frequency of $1.5 \mathrm{GHz}$. For the $7.5-\mathrm{mm}$ device, the EO bandwidth is greater than $67 \mathrm{GHz}$ (Fig. 4a), which is beyond the measurement limit of our vector network analyser (VNA). These voltage and bandwidth parameters indicate a much better performance over conventional LN modulators and are well suited for high-speed operation beyond 100 Gbaud. The input return losses $\left(\mathrm{S}_{11}\right.$ parameter) of both devices are less than $-18 \mathrm{~dB}$ at up to $67 \mathrm{GHz}$, which are small enough for practical use (Fig. 4b).

The fibre-to-fibre insertion losses at peak transmission are measured to be $8.6 \mathrm{~dB}$ and $8.25 \mathrm{~dB}$ for $13 \mathrm{~mm}$ and $7.5 \mathrm{~mm}$ device, respectively. The coupling loss of the grating couplers is $3.4 \mathrm{~dB} /$ facet. Therefore, the on-chip losses are $1.8 \mathrm{~dB}$ and $1.45 \mathrm{~dB}$ for $13 \mathrm{~mm}$ and $7.5 \mathrm{~mm}$ device, respectively. The coupling loss can be further improved by replacing grating coupler with edge-coupled spot-size converters, with fibre-to-fibre losses below $4 \mathrm{~dB}$ practically achievable.

Data modulation. We use our low- $V_{\pi}$, large-bandwidth, and lowloss 13-mm LNOI IQ modulators to generate advanced modulation formats of up to $320 \mathrm{~Gb} \mathrm{~s}^{-1}$. The experimental setup is 

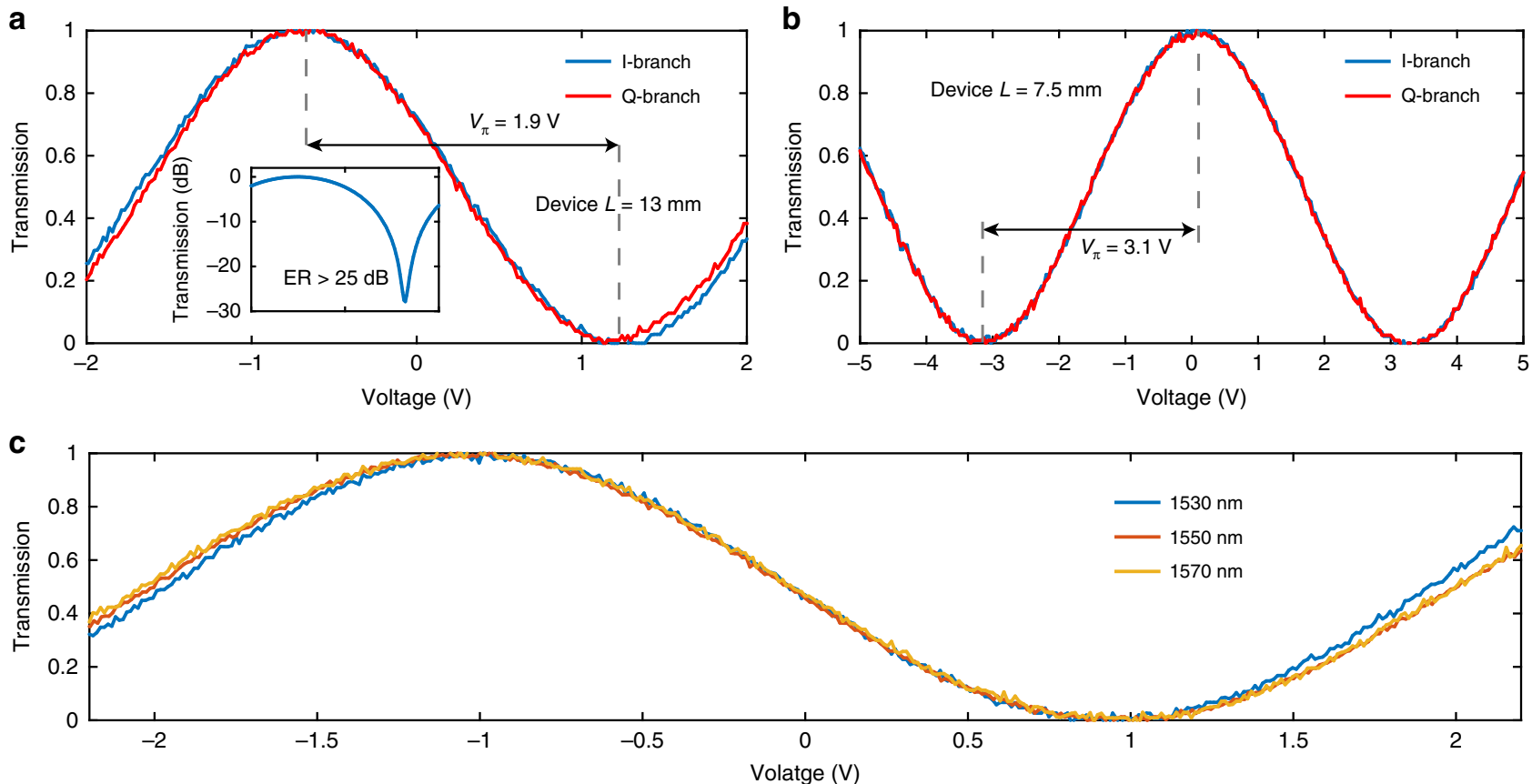

Fig. 3 Static EO characteristics. a, b Normalised optical transmission of both branches of the 13-mm and 7.5-mm devices as a function of the applied voltage, showing $V_{\pi}$ of $1.9 \mathrm{~V}$ and $3.1 \mathrm{~V}$, respectively. The inset of a shows the measured normalised transmission on a logarithmic scale, showing an extinction ratio greater than $25 \mathrm{~dB}$. c Measured optical transmission at different wavelengths.
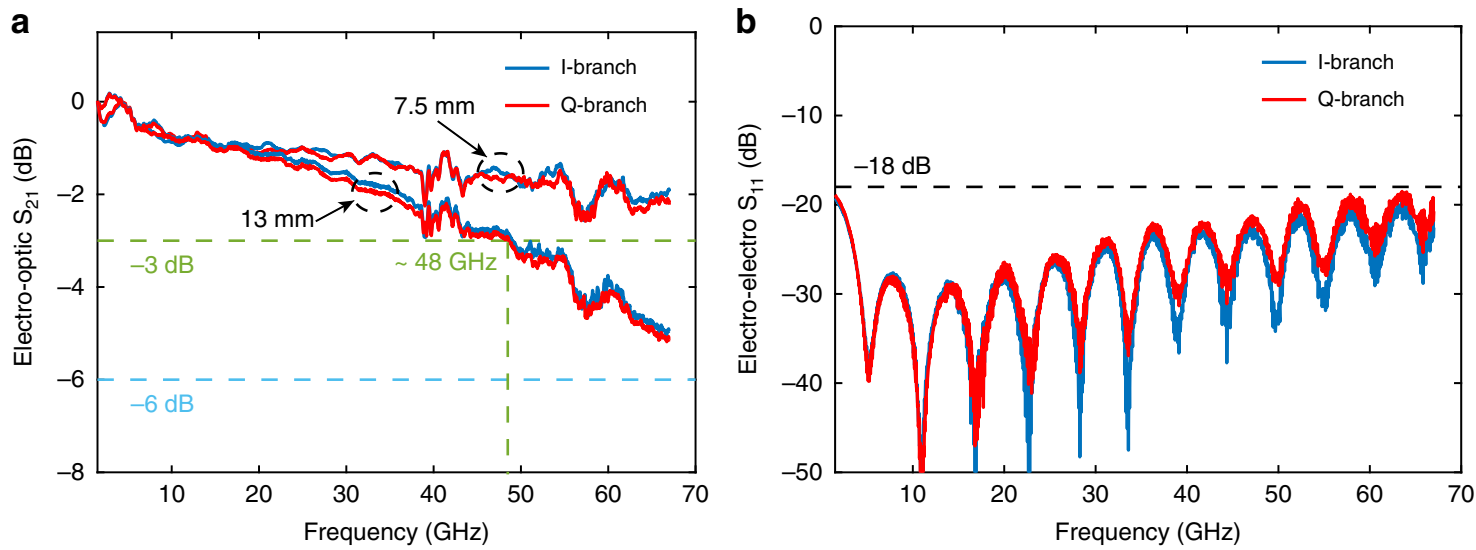

Fig. 4 Small-signal response. a EO bandwidths ( $S_{21}$ parameter) and $\mathbf{b}$ electrical reflection $S_{11}$ of the 13-mm IQ modulator.

shown in Fig. 5a. First, we demonstrate the generation of QPSK, one of the most widely deployed formats in coherent transmission systems, for performance benchmarking. Figure 5b-e summarises the measured constellation diagrams at $60,80,100$, and 110 Gbaud, corresponding to data-transmission rates of 120,160 , 200 , and $220 \mathrm{~Gb} \mathrm{~s}^{-1}$, respectively. All the modulated QPSK signals yield very good bit error rate (BER) performance well below the KP4 forward error correction (FEC) limit of $2 \times 10^{-4}$. Moreover, error-free operations at 80 and 100 Gbaud were achieved with BER $<1 \times 10^{-9}$. We then use 16 QAM modulation formats at high baud rate to further increase the data rates and investigate the signal-to-noise ratio (SNR), a common measure for the fidelity of modulated signals. The constellation diagrams at 60 and 80 Gbaud with QAM are shown in Fig. 5f, g. With the 60 Gbaud 16 QAM (data rate of $240 \mathrm{Gbit} \mathrm{s}^{-1}$ ), we can achieve a low BER of $8.6 \times 10^{-5}$. With the 80 Gbaud 16 QAM (data rate of $320 \mathrm{Gbit} \mathrm{s}^{-1}$ ), the measured BER of $8.4 \times 10^{-3}$ is still within the tolerance of the soft-decision forward error correction (SDFEC) limit of $4 \times 10^{-2}$. The back-to-back (B2B) BER curves at
60 Gbaud 16 QAM, shown in Fig. 5h. The BERs are also well below the SD-FEC limit and no error floor can be observed in the measurement. The high SNR demonstrated here benefits from a combination of linear EO response, low insertion loss and pure phase modulation, all of which are derived from the Pockels effect in the LN material.

\section{Discussion}

As presented above, the reported IQ modulator demonstrates ultrahigh speed and high-signal fidelity when encoding information into the amplitude and phase of the light. As far as we are aware, this is the first reported successful demonstration of IQ modulators based on LNOI platforms. The overall performance of optical modulators in terms of voltage, bandwidth, and footprint can be evaluated by two figures of merit: the $V_{\pi} L$ and $\mathrm{BW} / V_{\pi}$. The present device exhibits $V_{\pi} L$ and $\mathrm{BW} / V_{\pi}$ of around $2.5 \mathrm{~V} \mathrm{~cm}$ and $25 \mathrm{GHz} \mathrm{V}^{-1}$, significantly outperforming conventional LN counterparts. The overall length of the present device is $\sim 15 \mathrm{~mm}$, which is small enough to fit into compact coherent 
a
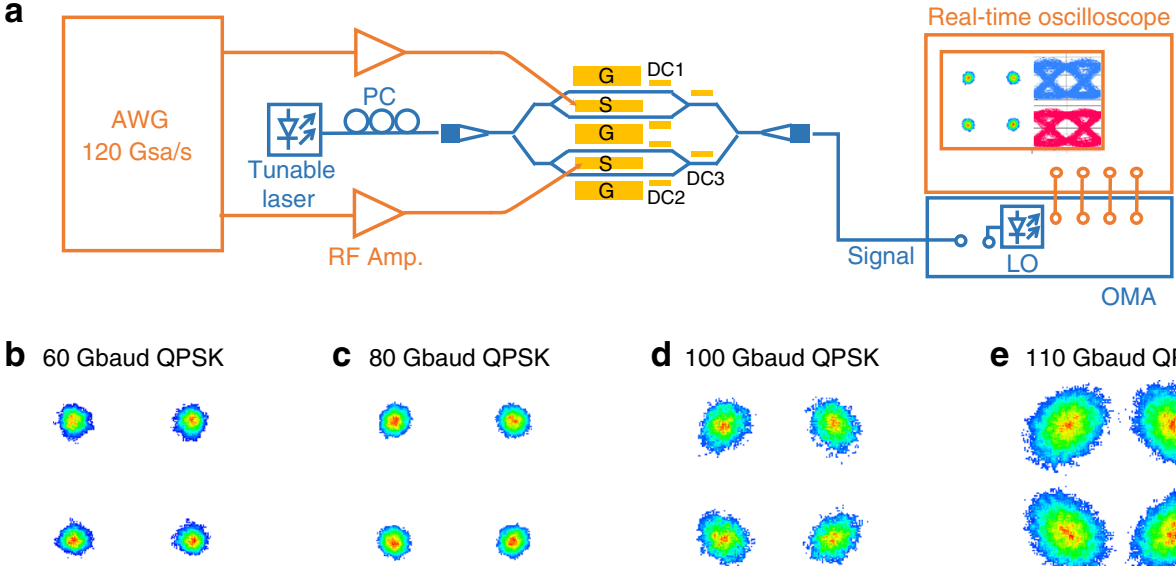

C 80 Gbaud QPSK

d 100 Gbaud QPSK

$\mathrm{BER}=2.52 \times 10^{-55}$
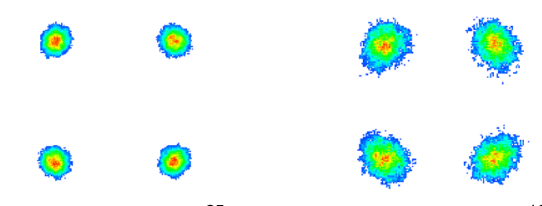

$\mathrm{BER}=1.56 \times 10^{-25}$

$\mathrm{BER}=4.67 \times 10^{-10}$

e 110 Gbaud QPSK

h
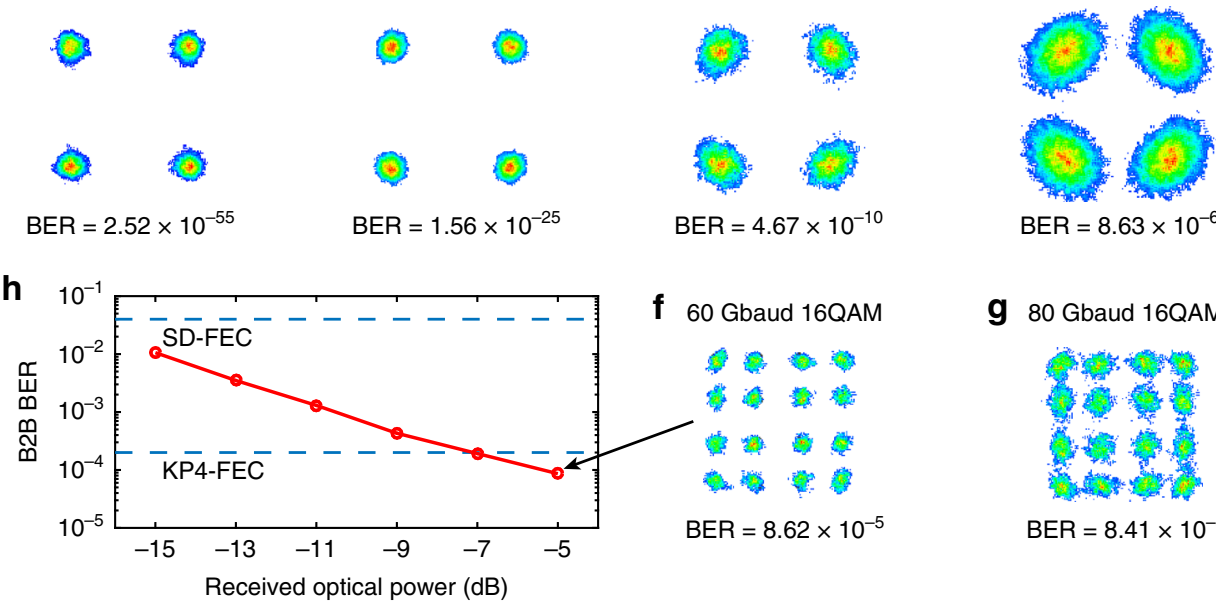

$\mathrm{BER}=8.63 \times 10^{-6}$

g 80 Gbaud 16QAM

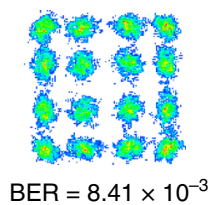

Fig. 5 Data modulation testing. a Experimental setup for coherent data transmission. AWG: arbitrary waveform generator, LO: local oscillator, OMA: optical modulation analyser, PC: polarisation controller. b-g Constellation diagram for QPSK signals with symbol rates of 60, 80, 100, and 110 Gbaud and 16 QAM signals with symbol rates of 60 and 80 Gbaud. $\mathbf{h}$ Measured curve of BER versus the received optical power for 60 Gbaud 16 QAM signal.

Table 1 Comparison of several performance metrics of IQ modulators.

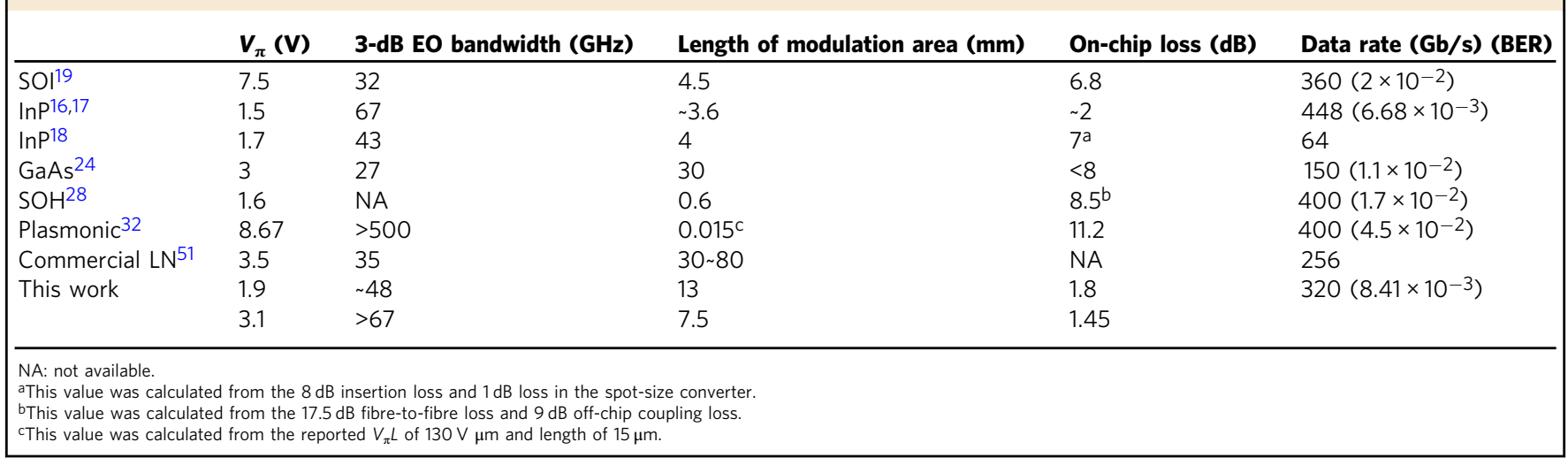

transceiver packages, such as the CFP2-ACO (C-form factor pluggable analogue coherent optics) and QSFP-DD (quad small form factor pluggable double density).

In Table 1, we compare the performance of the present device with the state-of-the-art, including commercial LN IQ modulators, and IQ modulators based on silicon, InP, GaAs, silicon-organichybrid $(\mathrm{SOH})$ and plasmonics. Conventional LN IQ modulators are also included as a benchmark. Clearly, the present device features the best optical loss compared among the others, and to the best of our knowledge, this is the lowest insertion loss ever achieved in IQ modulators. This characteristic makes the present device suitable for applications in short reach links, where the optical power budget becomes critical owing to prohibitive deployment of optical amplifiers. The $V_{\pi}$ and bandwidth of the present device are also very appealing for high-speed and lowpower consumption operations, which are comparable to those of the best InP modulators. Furthermore, in contrast to InP-based IQ modulators, in which thermoelectric cooling (TEC) is indispensable for reliable operation, the LNOI-based IQ modulator could be operated without TEC, which is highly desirable for lowcost applications. We believe that the EO bandwidth of the present device could be further extended beyond $100 \mathrm{GHz}$ without compromising the half-wave voltage, by further optimising the travelling-wave electrode (see Supplementary Note 4). This could support data rates of over 200 Gbaud. By further integrating on-chip polarisation combiners, a single LNOI-based modulator could operate at a data rate of $>1 \mathrm{~Tb} \mathrm{~s}^{-1}$ using, for example, 16 QAM modulation at 200 Gbaud. The demonstrated LNOI platform could therefore lead to a paradigm shift in building compact and high-performance IQ modulators, offering a crucial edge for future ultra-fast and low-power consumption optical fibre interconnects. 


\section{Methods}

Device fabrication. The devices were fabricated on a commercial X-cut LNOI wafer from NANOLN. We used electron-beam lithography (EBL) to define waveguide patterns after spinning Hydrogen silsesquioxane (HSQ). The $300-\mathrm{nm}$ high ridges of the LN waveguides were formed in an optimised argon plasma in an inductively coupled plasma etching system. Afterwards, we deposited a 220-nm amorphous-Si (a-Si) layer on the patterned LN waveguides and spin-coated HSQ on the a-Si for EBL. The Si/LN grating couplers were used for off-chip coupling. Then, we used a polymethyl methacrylate resist for a lift-off process to produce the 200-nm-thick TO phase shifters. Finally, 900-nm-thick gold travelling-wave electrodes were patterned through a lift-off process.

High-speed data modulation. At the transmitter, the two independent RF signals with a length of a pseudo-random bit sequence of $2^{15}-1$ were generated from a 120 -GSa/s arbitrary waveform generator (Keysight, M8194A). A root-raised-cosine filter was used for pulse shaping in the frequency domain. After being amplified by linear amplifiers (SHF S807C, $3 \mathrm{~dB}$ BW: $55 \mathrm{GHz}$ ), the output signals were fed into the IQ modulator by an RF probe (ground-signal-ground-signal-ground (GSGSG) configuration, $3 \mathrm{~dB} \mathrm{BW}>67 \mathrm{GHz}$ ). A second GSGSG RF probe was used to terminate the end of the transmission line with a $50-\Omega$ load to avoid back-reflected signals.

Light from a tunable laser (Santec TSL-550) with $17 \mathrm{dBm}$ output power was coupled into and collected from the chip via amorphous-Si/LN grating couplers. A polarisation controller was used to ensure transverse-electric mode input. An optical modulation analyser (OMA, Keysight N4391B) with a four-channel realtime oscilloscope (Keysight UXR0704A, $256 \mathrm{GSa} / \mathrm{s}$ ) served as a receiver. The modulated signal was received $\mathrm{B} 2 \mathrm{~B}$ and mixed with an internal local oscillator in the OMA. A series of digital signal processing steps, including low-pass filtering, polarisation demultiplexing, and feed-forward compensation, were included in the vector signal analysis (Keysight) software. The data decoded from the OMA were used for BER and error vector magnitude (EVM) measurements. The EVM describes the deviation of a measured symbol point from its ideal position in the constellation diagram and can be used to reliably estimate the BER values assuming the signal is distorted by additive white Gaussian noise only ${ }^{50}$.

Modulator energy considerations. For 16 QAM modulation, we can estimate the energy consumption per bit dissipated in the travelling-wave IQ modulator as $W_{\mathrm{bit}, 16 \mathrm{QAM}}=2 \times V_{\mathrm{rms}}{ }^{2} /(\mathrm{BR})$, where $V_{\mathrm{rms}}$ is the root-mean-square voltage of the electrical PAM4 signal, $B$ is the total bit rate and $R$ is the equivalent resistor of $50 \Omega$. The value of $V_{\mathrm{rms}}$ applied to the one MZM was measured directly using the oscilloscope. For $60 \mathrm{Gbaud} 16 \mathrm{QAM}\left(B=240 \mathrm{Gbit} \mathrm{s}^{-1}\right)$ modulation experiment with $V_{\text {rms }}=0.85 \mathrm{~V}$, we can calculate an energy consumption of $120 \mathrm{fJ} / \mathrm{bit}$. For 80 Gbaud 16 QAM $\left(B=320 \mathrm{Gbit} \mathrm{s}^{-1}\right)$ modulation experiment with $V_{\mathrm{rms}}=0.7 \mathrm{~V}$, we find a lower power consumption of $61 \mathrm{fj} / \mathrm{bit}$. To further reduce the energy consumption, we fabricated a LN MZM with a record low $V_{\pi}$ of $1.25 \mathrm{~V}$ while maintaining a high modulation bandwidth (see Supplementary Note 6 and Supplementary Fig. 7).

\section{Data availability}

All the data supporting the findings in this study are available in the paper and Supplementary Information. Additional data related to this paper are available from the corresponding authors upon request.

Received: 2 January 2020; Accepted: 16 July 2020;

Published online: 06 August 2020

\section{References}

1. Cisco, I. Cisco visual Networking Index: Forecast and Methodology 2015-2020 (Cisco, 2016)

2. Winzer, P. J. \& Neilson, D. T. From scaling disparities to integrated parallelism: a decathlon for a decade. J. Lightwave Technol. 35, 1099-1115 (2017).

3. Kikuchi, K. Fundamentals of coherent optical fiber communications. J. Lightwave Technol. 34, 157-179 (2016)

4. Winzer, P. J. et al. Spectrally efficient long-haul optical networking using 112-Gb/s polarization-multiplexed 16-QAM. J. Lightwave Technol. 28, 547-556 (2010).

5. Infinera Corporation. Coherent DWDM Technologies (Infinera Corporation, 2012).

6. Raybon, G. et al. High symbol rate coherent optical transmission systems: 80 and 107 Gbaud. J. Lightwave Technol. 32, 824-831 (2014).

7. Yamazaki, H., Sano, A., Nagatani, M. \& Miyamoto, Y. Single-carrier 1-Tb/s PDM-16QAM transmission using high-speed InP MUX-DACs and an integrated OTDM modulator. Opt. Express 23, 12866-12873 (2015).
8. Saida, T. Emerging integrated devices for coherent transmission-digitally assisted analog optics. In 2017 Optical Fiber Communications Conference and Exhibition (OFC) (IEEE, Los Angeles, 2017).

9. Ogiso, Y. et al. Ultra-high bandwidth InP IQ modulator co-assembled with driver IC for beyond 100-GBd CDM. In 2018 Optical Fiber Communication Conference (IEEE, San Diego, 2018).

10. Doerr, C. Coherent optics in Si photonics. In 2018 Optical Fiber Communication Conference (IEEE, San Diego, 2018).

11. Chen, A. \& Murphy, E. Broadband Optical Modulators: Science, Technology, and Applications (CRC Press, USA, 2011).

12. Wooten, E. L. et al. A review of lithium niobate modulators for fiber-optic communications systems. IEEE J. Sel. Top. Quantum Electron. 6, 69-82 (2000).

13. Raybon, G. et al. 180-GBaud All-ETDM single-carrier polarization multiplexed QPSK transmission over $4480 \mathrm{~km}$. In 2018 Optical Fiber Communication Conference (IEEE, San Diego, 2018).

14. Schuh, K. et al. Single carrier $1.2 \mathrm{Tbit} / \mathrm{s}$ transmission over $300 \mathrm{~km}$ with PM-64 QAM at 100 GBaud. In 2017 Optical Fiber Communication Conference (IEEE, Los Angeles, 2017).

15. Going, R. et al. Multi-channel InP-based coherent PICs with hybrid integrated SiGe electronics operating up to $100 \mathrm{GBd}, 32 \mathrm{QAM}$. In European Conference on Optical Communication (IEEE, Gothenbug, 2017).

16. Ogiso, Y. et al. Over $67 \mathrm{GHz}$ bandwidth and $1.5 \mathrm{~V} \mathrm{~V} \pi \mathrm{InP}$-based optical IQ modulator with n-i-p-n heterostructure. J. Lightwave Technol. 35, 1450-1455 (2017).

17. Ogiso, Y. et al. Ultra-high bandwidth InP IQ modulator for beyond 100-GBd transmission. In 2019 Optical Fiber Communication Conference (IEEE, San Diego, 2019).

18. Lange, S. et al. Low power InP-based monolithic DFB-laser IQ modulator with SiGe differential driver for 32-GBd QPSK modulation. J. Lightwave Technol. 34, 1678-1682 (2016)

19. Lin, J., Sepehrian, H., Rusch, L. A. \& Shi, W. Single-carrier 72 GBaud 32QAM and 84 GBaud 16QAM transmission using a SiP IQ modulator with joint digital-optical pre-compensation. Opt. Express 27, 5610-5619 (2019).

20. Samani, A. et al. $180 \mathrm{~Gb} / \mathrm{s}$ single carrier single polarization 16-QAM transmission using an O-band silicon photonic IQM. Opt. Express 27, 14447-14456 (2019).

21. Goi, K. et al. Low-loss high-speed silicon IQ modulator for QPSK/DQPSK in C and L bands. Opt. Express 22, 10703-10709 (2014).

22. Dong, P. et al. 112-Gb/s monolithic PDM-QPSK modulator in silicon. Opt. Express 20, B624-B629 (2012).

23. Dong, P. et al. Silicon in-Phase/quadrature modulator with on-chip optical equalizer. J. Lightwave Technol. 33, 1191-1196 (2015).

24. Schindler, P. C. et al. Monolithic GaAs electro-optic IQ modulator demonstrated at $150 \mathrm{Gbit} / \mathrm{s}$ with 64QAM. J. Lightwave Technol. 32, 760-765 (2014).

25. Lauermann, M. et al. Low-power silicon-organic hybrid ( $\mathrm{SOH})$ modulators for advanced modulation formats. Opt. Express 22, 29927-29936 (2014).

26. Korn, D. et al. Silicon-organic hybrid (SOH) IQ modulator using the linear electro-optic effect for transmitting 16QAM at $112 \mathrm{Gbit} / \mathrm{s}$. Opt. Express 21, 13219-13227 (2013).

27. Leuthold, J. et al. Silicon-organic hybrid electro-optical devices. IEEE J. Sel. Top. Quantum Electron 19, 114-126 (2013).

28. Wolf, S. et al. Coherent modulation up to $100 \mathrm{GBd}$ 16QAM using siliconorganic hybrid (SOH) devices. Opt. Express 26, 220-232 (2018).

29. Haffner, C. et al. All-plasmonic Mach-Zehnder modulator enabling optical high-speed communication at the microscale. Nat. Photon 9, 525-528 (2015).

30. Haffner, C. et al. Low-loss plasmon-assisted electro-optic modulator. Nature 556, 483-486 (2018).

31. Messner, A. et al. Plasmonic ferroelectric modulators. J. Lightwave Technol. 37, 281-290 (2019)

32. Heni, W. et al. Plasmonic IQ modulators with attojoule per bit electrical energy consumption. Nat. Commun. 10, 1694 (2019).

33. Janner, D. et al. Micro-structured integrated electro-optic $\mathrm{LiNbO}_{3}$ modulators. Laser Photon Rev. 3, 301-313 (2009).

34. Poberaj, G., Hu, H., Sohler, W. \& Günter, P. Lithium niobate on insulator (LNOI) for micro-photonic devices. Laser Photon Rev. 6, 488-503 (2012).

35. Guarino, A. et al. Electro-optically tunable microring resonators in lithium niobate. Nat. Photon 1, 407-410 (2007).

36. Jin, $\mathrm{S}$., Xu, L., Zhang, H. \& Li, Y. $\mathrm{LiNbO}_{3}$ Thin-film modulators using silicon nitride surface ridge waveguides. IEEE Photonics Technol. Lett. 28, 736-739 (2016).

37. Rao, A. et al. High-performance and linear thin-film lithium niobate Mach-Zehnder modulators on silicon up to $50 \mathrm{GHz}$. Opt. Lett. 41, 5700-5703 (2016).

38. Cai, L., Kang, Y. \& Hu, H. Electric-optical property of the proton exchanged phase modulator in single-crystal lithium niobate thin film. Opt. Express 24, 4640-4647 (2016). 
39. Chang, L. et al. Heterogeneous integration of lithium niobate and silicon nitride waveguides for wafer-scale photonic integrated circuits on silicon. Opt. Lett. 42, 803-806 (2017).

40. Wang, C. et al. Integrated lithium niobate electro-optic modulators operating at CMOS-compatible voltages. Nature 562, 101-104 (2018).

41. Boes, A. et al. Status and potential of lithium niobate on insulator (LNOI) for photonic integrated circuits. Laser Photon. Rev. 12, 19 (2018).

42. He, M. et al. High-performance hybrid silicon and lithium niobate Mach-Zehnder modulators for 100 Gbit s-1 and beyond. Nat. Photon. 13, 359-364 (2019).

43. Mercante, A. J. et al. Thin film lithium niobate electro-optic modulator with terahertz operating bandwidth. Opt. Express 26, 14810-14816 (2018).

44. Rao, A. \& Fathpour, S. Compact lithium niobate electrooptic modulators. IEEE J. Sel. Top. Quantum Electron 24, 14 (2018).

45. Chen, L., Xu, Q., Wood, M. G. \& Reano, R. M. Hybrid silicon and lithium niobate electro-optical ring modulator. Optica 1, 112-118 (2014).

46. Weigel, P. O. et al. Bonded thin film lithium niobate modulator on a silicon photonics platform exceeding $100 \mathrm{GHz} 3-\mathrm{dB}$ electrical modulation bandwidth. Opt. Express 26, 23728-23739 (2018).

47. Jian, J. et al. High-efficiency hybrid amorphous silicon grating couplers for sub-micron-sized lithium niobate waveguides. Opt. Express 26, 29651-29658 (2018).

48. Nagata, H., Brien, N. F. O., Bosenberg, W. R., Reiff, G. L. \& Voisine, K. R. DCvoltage-induced thermal shift of bias point in $\mathrm{LiNbO}_{3}$ optical modulators. IEEE Photonics Technol. Lett. 16, 2460-2462 (2004).

49. Salvestrini, J. P., Guilbert, L., Fontana, M., Abarkan, M. \& Gille, S. Analysis and control of the DC drift in $\mathrm{LiNbO}_{3}$-based Mach-Zehnder modulators. $J$. Lightwave Technol. 29, 1522-1534 (2011).

50. Schmogrow, R. et al. Error vector magnitude as a performance measure for advanced modulation formats. IEEE Photonics Technol. Lett. 24, 61-63 (2012).

51. Fujitsu Optical Components Limited. 100G/400G LN modulator, Fujitsu https://www.fujitsu.com/jp/group/foc/en/products/optical-devices/ 100gln/index.html (2020).

\section{Acknowledgements}

This work was supported by the National Key R\&D Program of China (Grant Nos. 2019YFA0705000, 2019YFB1803900), the National Natural Science Foundation of China (Grant Nos. 11690031, 11761131001), the Key R\&D Program of Guangdong Province (Grant No. 2018B030329001), the Local Innovative and Research Teams Project of Guangdong Pearl River Talents Program (Grant No. 2017BT01X121), the Innovation Fund of WNLO (Grant No. 2018WNLOKF010) and the Project of Key Laboratory of Radar Imaging and Microwave Photonics, Ministry of Education (Grant No. RIMP2019003).

\section{Author contributions}

X.C., M.X., and X.X. conceived device design. M.H., J.J., and and P.Y. carried out the LN fabrication. M.X., H.Z., and Z.L. carried out the measurement. S.H.Y., S.Y.Y., X.X., and X.C. carried out the data analysis. All authors contributed to the writing. X.C. finalized the paper. S.Y.Y., S.H.Y., X.X., and X.C. supervised the project.

\section{Competing interests}

H.Z., X.X., and S.H.Y. are involved in developing silicon photonics technologies at China Information and Communication Technologies Group Corporation. The remaining authors declare no competing interests.

\section{Additional information}

Supplementary information is available for this paper at https://doi.org/10.1038/s41467020-17806-0.

Correspondence and requests for materials should be addressed to X.X. or X.C.

Peer review information Nature Communications thanks Arnan Mitchell and the other, anonymous, reviewers for their contribution to the peer review of this work. Peer reviewer reports are available.

Reprints and permission information is available at http://www.nature.com/reprints

Publisher's note Springer Nature remains neutral with regard to jurisdictional claims in published maps and institutional affiliations.

pen Access This article is licensed under a Creative Commons Attribution 4.0 International License, which permits use, sharing, adaptation, distribution and reproduction in any medium or format, as long as you give appropriate credit to the original author(s) and the source, provide a link to the Creative Commons license, and indicate if changes were made. The images or other third party material in this article are included in the article's Creative Commons license, unless indicated otherwise in a credit line to the material. If material is not included in the article's Creative Commons license and your intended use is not permitted by statutory regulation or exceeds the permitted use, you will need to obtain permission directly from the copyright holder. To view a copy of this license, visit http://creativecommons.org/ licenses/by/4.0/.

(C) The Author(s) 2020 